JHU-TIPAC-920027/rev

May, 1993

\title{
Astrophysical Limit on the Deformation of the Poincaré Group
}

\author{
G. Domokos and S. Kovesi-Domokos' \\ The Henry A. Rowland Department of Physics and Astronomy \\ The Johns Hopkins University \\ Baltimore, MD 21218
}

\begin{abstract}
The deformed Poincaré group contains a characteristic mass, $\kappa$. At energies exceeding $\kappa$, deviations from the special theory of relativity become significant. However, small deviations from ordinary relativistic kinematics are observable even at energies substantially lower than $\kappa$. The observation of distant events producing ultra high energy (UHE) particles leads to a lower limit on $\kappa$. From an analysis of the UHE data on the burst in the binary system HER X1 in 1986, we deduce $\kappa \gtrsim 10^{12} \mathrm{GeV}$.
\end{abstract}

\footnotetext{
${ }^{1}$ E-mail: SKD@JHUP.PHA.JHU.EDU
} 
The special theory of relativity is one of the fundamental ingredients of any calculation or experiment in particle physics. Therefore, it is important to establish the accuracy to which it can be trusted.

This is a non-trivial problem. Practically all analyses of current particle physics experiments assume the validity of the special theory of relativity, i.e. the invariance of local physics under the Poincaré group: hence, by design, they cannot be used to test Poincaré invariance, unless, perhaps, that invariance fails in a dramatic and truly unexpected way. As a consequence, while one knows that the special theory of relativity is not applicable on length scales where gravitational effects are important, its validity at short distances is harder to test.

It has been proposed several times that Poincaré invariance is violated at short distances; for some recent proposals see [1]. However, most of the proposals were introduced in an ad hoc fashion; the internal consistency of the schemes has never been investigated systematically. (There also exists a substantial number of older works in this area, involving a variety of ideas, such as the discretization of space-time, etc. ; it is not our purpose to review them here.)

Due to developments in the theory of group deformations, the situation has changed, however. In some recent papers, [2], Lukierski et. al. developed a theory of the deformation of the Poincaré group. In order to accomplish the task, the authors of ref. [2] started from the DeSitter group. They performed a group deformation along the lines described in the classic papers on the deformation of simple groups, [3], [4, [5] and afterwards they performed a contraction to a deformed Poincaré group. While the procedure referred to is not a unique one, (see, e.g. [6]) it is internally consistent. Moreover, it is attractive from the physical point of view: the theory contains a characteristic mass, $\kappa$. The latter determines the energies below which the special theory of relativity is valid to a good approximation; at energies above $\kappa$, significant deviations from the familiar relativistic kinematics are to be expected. The important point is that on can obtain useful information about the possible deformation of the Poincaré group from the kinematics of a single particle alone. (This is due to the fact that the translation subgroup remains an Abelian one: the rules of the addition of momenta are unchanged.)

Existing and future observational data on ultra high energy (UHE), distant events can place an interesting lower limit on the characteristic mass, $\kappa$. For this purpose one needs, in essence, only one of the results developed in refs. ([2]). In those papers it was shown that the quadratic Casimir invariant of the Poincaré group is deformed to the following expression:

$$
C_{\kappa}=-\left(\mathbf{p}^{2}+2 \kappa^{2}\left(\cos \frac{E}{\kappa}-1\right)\right) .
$$

In eq. (11) the quantities $\mathbf{p}$ and $E$ have their usual meaning. The deformed Casimir invariant, eq. (国) goes over to the familiar quadratic form as $\kappa \rightarrow \infty$. However, at any $\kappa<\infty$, there is an observable difference between ordinary relativistic kinematics and the one described by eq. (四). Let us consider, in particular, a 
single particle state, defined, as usual, as an irreducible representation of the deformed Poincaré group. In that case, $C_{\kappa}=m^{2}$, where $m$ is related to the the rest energy of the particle. On putting $\mathbf{p}=0$, the rest energy is given by:

$$
E^{0}=\kappa \cos ^{-1}\left(1-\frac{m^{2}}{2+\kappa^{2}}\right),
$$

which agrees with the usual expression up to corrections of $O\left(m^{4} / \kappa^{4}\right)$. One can compute the group velocity $(v)$ of the particle in the deformed Poincare group. As whithin the framework of the usual (undeformed) Poincaré group, if the energy of the particle is much greater than $m$, the latter can be neglected. In this approximation one has:

$$
\frac{1}{v}=\frac{d|\mathbf{p}|}{d E} \approx \cos \frac{E}{2 \kappa} .
$$

The deformed Poincaré group leads to the result that a massless particle is, in the usual sense of the word, always superluminal: what one accepts these days as the "speed of light" is only the low frequency limit of the group velocity.

One can now place a lower limit on the value of $\kappa$ as follows. Suppose that a periodic UHE signal emitted by a distant source, (e.g. a pulsar) is observed.

The time delay a $\delta$ function pulse suffers between a source a distance $d$ away from a (terrestrial) detector is given by the elementary formula:

$$
\Delta t=\frac{d}{v}=d \cos \left(\frac{E}{2 \kappa}\right)
$$

We represent the signal by means of a Fourier series. For a signal of fundamental period $T$ and energy $E$, one has at the source:

$$
I\left(E, t_{s}\right)=\sum_{n=-\infty}^{\infty} I_{n}^{S}(E) \exp i n \omega t_{s},
$$

where $\omega=2 \pi / T$. The intensity is real, $I_{-n}=I_{n}^{*}$. At the detector, the Fourier coefficients differ from the ones at the source by an energy dependent phase shift, analogously to the time delay in eq. (田):

$$
I_{n}^{D}(E)=I_{n}^{S}(E) \exp \left[i \omega n d \cos \left(\frac{E}{2 \kappa}\right)\right]
$$

We now average eq. (6) over the energies, assuming, as usual that the spectrum of the source is given by a power law:

$$
\frac{d \Phi}{d E}=C E^{-\alpha}
$$

where $C$ is a normalization constant in order to assure $\int_{E_{\min }}^{E_{\max }}(d \Phi / d E) d E=1$. Here, the quantities $E_{\min }$ and $E_{\max }$ stand for the minimal and maximal energies 
observed. The averaging over energies is made necessary by the fact that one has no direct information on the pulse shape at the source. We have:

$$
\left\langle I_{n}\right\rangle=C \int_{E_{\min }}^{E_{\max }} I_{n}^{S}(E) E^{-\alpha} \exp \left[i \omega n d \cos \left(\frac{E}{2 \kappa}\right)\right]
$$

As a consequence of the velocity dispersion, both the magnitude and the phase of $\left\langle I_{n}^{D}\right\rangle$ differ from $I_{n}^{S}$. On assuming that the pulse at the source is proportional to a $\delta$ function at all energies - i.e. that the entire observed width of the pulse arises from velocity dispersion - one can obtain a plausible lower limit on $\kappa$. (Evidently, this assumption means that $I_{n}^{S}$ is independent of $n$ and E.) The argument goes as follows.

Assuming that the pulse is a $\delta$ function at the source at any energy, one shows in a straight-forward manner that the signal observed at the detector has a finite width as a consequence of the assumed velocity dispersion: one just has to insert $I_{n}^{S}=I=$ const into eq. (8). The reason why such an argument cannot be formulated as a rigorous theorem is that one can construct counterexamples showing that a signal emitted at a source can actually become narrower at arrival. (For instance, one can imagine that the high energy particles are emitted after the low energy ones and just catch up with them at the detector. Thus the pulse observed at the detector is narrower than the one at the source. Examples of this kind have been analyzed in detail by [7].) One has to note, nevertheless that all scenarios of the type mentioned above are highly "unnatural": they require a delicate "fine tuning" of the pulse at the source according to its distance from a detector at Earth. (In particular, there is no known acceleration mechanism which would produce effects similar to the one described above.) For this reason, we do not consider mechanisms of this type any further. In order to complete the argument, we note that the pulse has a finite natural width at the source. Hence, any broadening caused by a velocity dispersion is superposed onto the natural width. Consequently, even in the total absence of a velocity dispersion, one would see a finite width and, hence, estimate that $\kappa<\infty$.

The unknown coefficient $I$ is eliminated by taking ratios of the moduli of the Fourier coefficients. It is evident from these equations that in order to obtain good lower limits on $\kappa$,

- one has to maximize the distance between the source and the detector,

- one has to use data at the highest energies available.

There was a burst observed in the binary system HER-X1 in 1986. The distance of this system is about $5 \mathrm{kpc}$; particles of the highest energies were observed by the CYGNUS collaboration by means of detecting extensive air showers induced by them, $c f$. ref. [B]. The nominal threshold of the detector as stated in ref. [8] is $70 \mathrm{TeV}$. Using the period-folded data from this experiment and the published fundamental period, $(T \approx 1.24 \mathrm{sec})$, ref. [9], we determined the first few Fourier coefficients from the data, [10]. 
(Due to the fact that 11 showers were observed by the CYGNUS collaboration, the data allow for a meaningful determination of the first five complex Fourier coefficients. The coefficient $I_{0}$, corresponding to a constant intensity, can always be subtracted.) The result is displayed in Table 1.

Table 1 : Fourier coefficients
\begin{tabular}{|c|c|c|}
\hline \hline$n$ & $\left|I_{n}^{D}\right|^{2}$ & $\arg I_{n}^{D}$ \\
\hline 1 & 0.99 & 0.044 \\
2 & 0.96 & 0.088 \\
3 & 0.92 & 0.13 \\
4 & 0.86 & 0.17 \\
5 & 0.79 & 0.21 \\
\hline
\end{tabular}

One observes that the phases of the Fourier coefficients determined from the data exhibit no noticeable trend; probably, they are considerably affected by "noise", i.e. by the various errors in the data set. This is a general phenomenon [11]. For this reason, we used only the moduli of the Fourier coefficients in order to obtain a lower limit on $\kappa$. (One determines by inspection that the moduli contain less noise than the phases: they are decreasing almost linearly with $n$.)

Using the ratio, $\left|\left\langle I_{2}^{D}\right\rangle /\left\langle I_{1}^{D}\right\rangle\right|^{2}$ from eq. (8), one can compute $\kappa$. As stated before, this is a lower limit on its value. The calculation cannot be carried out in closed form: for values of $\kappa, E_{\min }$ and $E_{\max }$ of interest, the integral cannot be well approximated by any closed expression in terms of known functions. On taking the minimal and maximal energies from ref. [8], the integral was computed numerically for various values of $\kappa$. Then, taking the the first four ratios of the moduli of the observed Fourier coefficients, the "optimal" value of $\kappa$ was determined by minimizing $\chi^{2}$ between the observed and computed values.

The function $\chi^{2}(\kappa)$ has a rather broad minimum at

$$
\kappa \approx 1.3 \times 10^{12} \mathrm{GeV} .
$$

Due to the fact that the determination of the primary energy of a shower is uncertain to about a factor of 2 (cf. [8]), it is of interest to test the sensitivity of the value (more precisely, the lower limit) of $\kappa$ to the primary energy. Inspection of eq. (8) shows that the the integral is more sensitive to variations of $E_{\max }$ than to $E_{\min }$. Indeed, varying $E_{\min }$ by a factor of 5, we found no sensitivity (to within $3 \%$ ) of the lower limit of $\kappa$. In Fig.(1), we plotted $\chi^{2}$ as a function of $E_{\max }$ and $\kappa$. It is evident that neither the lower limit of $\kappa$, nor the value of $\chi^{2}$ at minimum depend sensitively on the primary energy of the most energetic shower. In arriving at the result eq. (9), we used a value, $\alpha=1.4$, obtained from a rough fit to the data. 
The quoted lower limit is rather insensitive to the precise value of the spectral index.

Previous to our work, the authors of ref. [9] quoted a lower limit on the characteristic mass scale(s) of the ad hoc modifications of relativistic kinematics, [1]. They find a result which is equivalent in the present formulation to $\kappa \gtrsim 510^{11} \mathrm{GeV}$ ? The improvement represented by eq. (9) is due to the fact that by computing Fourier coefficients, one uses a larger amount of information contained in the data than by using the simpler estimates in ref. [9]. In fact, one sees from Fig.(1) that the value of $\kappa$ quoted in ref. [9] gives a $\chi^{2}$ about 3 times as high as its minimal value. More importantly, the the formula used in ref. [9], depends quadratically on $E_{\max }$ and, since $E_{\min } \ll E_{\max }$, it is practically independent of the least energetic shower. Thus, in effect, the lower limit is determined by a single event only. By contrast, in the method described here, not only the function $\chi^{2}(\kappa)$, but individual Fourier coefficients as well, show a much weaker dependence on the energy of an individual shower. Consequently, even if one or a few of the showers came from the background, the estimate of the lower limit will not be changed significantly.

It appears that deviations from Poincaré invariance are negligible at least up to energies which are fairly close to a presumed grand unification mass. Presumably, as larger data samples become available, this limit can be further improved, or else, one may be able to estimate the energy where the special theory of relativity breaks down due to some new physics. UHE astrophysical data are unique in this respect due to the high energies and large distances involved.

\section{Acknowledgement}

This research has been supported in part by the U.S. Department of Energy under Grant \# DE-FG-02-85ER40211. We also thank Alex Szalay for useful discussions.

\section{References}

[1] T.G. Pavlopoulos, Phys. Rev. 159 (1967) 1106; K. Fujiwara, Found. Phys. 10 (1980) 309.

[2] J. Lukierski, H. Ruegg, A. Nowicki and V.N. Tolstoy, Phys. Lett. 264B (1991) 331; J. Lukierski, A. Nowicki and H. Ruegg, Boston University preprint BUHEP-91-21 (1991).

[3] V.G. Drinfeld, "Quantum groups", Proc. Internat. Congress of Mathematics. University of California, Berkeley (1986); p. 793.

\footnotetext{
${ }^{2}$ In order to compare the estimate used in ref. [9] with ours, one has to approximate $\cos E / \kappa$ in eq. (4) by the first two terms of its Taylor series.
} 
[4] Yu.T. Manin, "Quantum groups and non-commutative geometry" Univ. of Montreal press, (1989).

[5] M. Jimbo, Lett. Math. Phys. 10 (1985) 63; ibid. 11 (1986) 247.

[6] O. Ogievetskii, private communication.

[7] J.R. Cudell, F. Halzen and P. Hoyer, Phys. Rev. D 36 (1987) 1637.

[8] CYGNUS collaboration, B.L. Dingus et al., Phys. Rev. Letters 61 (1988) 1906.

[9] CYGNUS collaboration, T.J. Haines et al., Phys. Rev. D41 (1990) 692.

[10] G. Domokos, B. Elliott and S. Kovesi-Domokos, Johns Hopkins University preprint JHU-TIPAC-920024 (1992).

[11] A. Szalay, private communication. 


\section{Figure Caption}

Fig.(1) The quantity $\chi^{2}$ is plotted as a function of $\kappa$ end of the maximal observed shower energy. $E_{\max }$ is plotted in $\mathrm{PeV} ; \kappa$ is plotted in units of $5 \times$ $10^{11} \mathrm{GeV}$. 